\title{
Diversité et état de conservation des espèces ligneuses alimentaires à la périphérie de la Forêt Classée de la Lama (Bénin)
}

\author{
Bidossèssi Eliane Juliette ASSOGBADJO ${ }^{1,2^{*}}$, Achille HOUNKPEVI ${ }^{1}$, \\ Yao Sadaiou Sabas BARIMA ${ }^{2}$, Ghislain Comlan AKABASSI ${ }^{3}$, Elie Antoine PADONOU ${ }^{4}$, \\ Yao Charles SANGNE ${ }^{2}$, Achille Ephrem ASSOGBADJO ${ }^{1,3}$ et Romain GLELE KAKAÏ ${ }^{1}$ \\ ${ }^{1}$ Laboratoire de Biomathématiques et d'Estimations Forestières (LABEF), Faculté des Sciences Agronomiques \\ (FSA), Université d'Abomey-Calavi (UAC), 04 BP 1525, Cotonou, Bénin. \\ ${ }^{2}$ Unité de Formation et de Recherche en Environnement (UFRE), Université Jean Lorougnon Guédé (UJLoG), \\ BP 150, Daloa, Côte d'Ivoire. \\ ${ }^{3}$ Laboratoire d'Ecologie Appliquée (LEA), Faculté des Sciences Agronomiques (FSA), Université d'Abomey- \\ Calavi (UAC), 01 BP 526, Cotonou Bénin. \\ ${ }^{4}$ Ecole de Foresterie Tropicale (EFT), Université Nationale d'Agriculture du Benin (UNA) Kétou, Bénin \\ *Auteur correspondant ; E-mail : assogbadjoeliane@gmail.com ; Tel : (00 229) 94155573
}

\author{
Received: 24-06-2021 \\ Accepted: 27-11-2021 \\ Published: 31-12-2021
}

\section{RESUME}

Les systèmes agroforestiers contribuent comme les forêts à la conservation physique et chimique du sol, à la régulation thermique et à la conservation de la biodiversité. Cette étude visait à caractériser la communauté des espèces ligneuses alimentaires dans quatre systèmes agroforestiers périphériques au noyau central de la Forêt Classée de la Lama. Au total, 770 placeaux carrés $(30 \mathrm{~m}$ x $30 \mathrm{~m}$ ) ont été installés de façon aléatoire dans les champs, plantations privées, jardins de case et jachères. Dans chaque placeau, des mesures dendrométriques (diamètre) ont été effectuées sur les individus de diamètre $\geq 5 \mathrm{~cm}$ à $1,30 \mathrm{~m}$ du sol. L'indice de similarité de Jaccard et le diagramme de Venn ont permis d'évaluer la similarité entre les systèmes agroforestiers. La valeur la plus élevée de l'indice de Shannon a été enregistrée dans les jachères. La structure en diamètre des ligneux présentait une prédominance des sujets de petit diamètre [5-25 cm [. Les jardins de case étaient les seuls regroupant des individus de petites tailles. Une faible variation floristique a été observée entre les systèmes. Il devient impérieux d'intervenir afin d'assurer la conservation de ces espèces.

(C) 2021 International Formulae Group. All rights reserved.

Mots clés : Diversité, systèmes agroforestiers, plantes alimentaires locales, Forêt de la Lama, Bénin.

\section{Diversity and conservation status of woody food species at the periphery of the Lama forest reserve (Benin)}

\section{ABSTRACT}

Agroforestry systems, like forests, contribute to the physical and chemical conservation of the soil, to thermal regulation and to the biodiversity conservation. The study aimed to characterize the community of edible woody species in four agroforestry surrounding the central core of the Lama Forest Reserve. A total of 770 square 
plots $(30 \mathrm{~m} \times 30 \mathrm{~m})$ were randomly installed in fields, private plantations, home gardens and fallows. In each plot, dendrometric measurements (diameter) were taken on individuals with a diameter $\geq 5 \mathrm{~cm}$ at $1.30 \mathrm{~m}$ from the ground. Jaccard similarity index and Venn diagram were used to assess the similarity between agroforestry systems. Species density varied significantly from fields to home gardens. The diameter structure of the woody plants showed a predominance of small diameter individuals [5-35 $\mathrm{cm}$ [. The home gardens were the only ones gathering individuals of small sizes. A low floristic variation was observed between the systems. It becomes imperative to intervene to ensure the conservation of these species.

(C) 2021 International Formulae Group. All rights reserved.

Keywords: Diversity, agroforestry systems, Edible woody species, Lama Forest, Benin.

\section{INTRODUCTION}

La population africaine de 1,2 milliard d'habitants en 2015 devrait atteindre 2,4 milliards en 2050 selon les projections des Nations Unies (Nations Unies, 2016). L'alimentation de cette population en croissance nécessite des systèmes de productions alimentaires optimisés et une productivité accrue (Phalan et al., 2011). En effet, pour satisfaire les besoins de la population africaine grandissante, l'accent est de plus en plus mis sur la promotion des systèmes agroforestiers qui sont des systèmes diversifiés et écologiquement durables (Saj et al., 2013). L'agroforesterie désigne l'ensemble des pratiques agricoles associant les arbres avec les cultures ou avec l'élevage pour augmenter les rendements de manière naturelle en utilisant au maximum les fonctionnalités écologiques et biologiques des écosystèmes (Suarez et al., 2012). Les systèmes agroforestiers offrent une large gamme de produits et de services par rapport aux systèmes de monoculture (Badiane et al., 2019). Ils constituent en effet une source importante de nombreux produits destinés à l'alimentation, au fourrage du bétail, à la médecine, au chauffage et à divers autres services pour les populations locales. Ces produits sont parfois dotés d'une signification sociale, culturelle ou religieuse (Vodouhê et al., 2008). En plus des diverses fonctions écologiques, l'association des arbres avec les plantes cultivées joue également le rôle de régulateur thermique par leur ombrage en créant un microclimat propice au bon développement des cultures (Diedhou et al., 2014).

Les systèmes agroforestiers basés sur des cultures pérennes représentent une part importante des zones agroforestières mondiales et impliquent un nombre considérable d'agriculteurs familiaux (Pedelahore, 2014). Au Bénin, le système agroforestier traditionnel est caractérisé par la présence d'espèces naturelles telles que Vitellaria paradoxa C.F.Gaertn (karité), Parkia biglobosa (Jacq.) R.Br. ex Benth (néré), Adansonia digitata L., (baobab) Tamarindus indica L., (tamarin), Afzelia africana Sm., (lingué) au Nord et les espèces ligneuses comme Elaeis guineensis Jacq., (palmier), Irvingia gabonensis (Aubry-Lecomte ex O'Rorke) Baill. (pommier), Triplochiton scleroxylon K.Schum., (samba), Milicia excelsa (Welw.) C.C.Berg, (iroko) au Sud (Biaou et al., 2016). Ces espèces sont épargnées pendant les défrichements en raison de leur valeur nutritionnelle et de leur participation à l'économie des ménages (Wala et al., 2005).

Cependant, la valeur nutritionnelle élevée et la facilité d'accès de ces essences dans les systèmes agroforestiers conduisent à leur surexploitation (Rigg, 2009). Par exemple, les individus d'espèces de plantes alimentaires dans les champs, les jachères ou les forêts classées sont fréquemment émondés pour l'alimentation humaine et du bétail et leur feuillage est vendu en zone urbaine (Ouedraogo et al., 2011). De plus, l'expansion et l'intensification des activités agricoles et l'urbanisation contribuent également à la forte dégradation de ces ressources (Akouehou et al., 2014).

Située au sud du Bénin, la forêt classée de la Lama (FCL) présente un intérêt particulier à cause de sa structure végétale (Ginisty, 2008). Elle fait partie des aires 
protégées représentant les derniers refuges pour les espèces ligneuses alimentaires au Sud du Bénin (Hermans et al., 2004). La FCL fait partie également des forêts sous fortes pressions anthropiques compte tenu de sa localisation dans une zone fortement peuplée (Toyi et al., 2017). Malgré le nombre important des travaux scientifiques menés dans cet écosystème, aucune initiative de recherche ne s'est intéressée aux espèces ligneuses alimentaires dans les systèmes agroforestiers de la zone périphérique du noyau central de la forêt classée de la Lama. Il est donc important d'étudier la diversité au sein de ces systèmes agroforestiers afin de mieux appréhender leur potentiel dans la conservation de la diversité végétale. Ainsi, cette étude visait à évaluer la diversité des plantes alimentaires locales et l'effet de l'intensité d'exploitation sur leur abondance dans quatre systèmes agroforestiers traditionnels de la périphérie du noyau central de la Forêt Classée de la Lama.

\section{MATERIEL ET METHODES \\ Zone d'étude}

Située au sud du Bénin et à une centaine de kilomètres de la côte, la Forêt Classée de la Lama (FCL) s'étend entre les latitudes $6^{\circ} 56^{\prime}$ et $7^{\circ} 00^{\prime}$ nord et entre les longitudes $2^{\circ} 08^{\prime}$ et $2^{\circ} 15^{\prime}$ est (Figure 1). Elle est gérée par l'Office National du Bois (ONAB) de la République du Bénin. Cette forêt est subdivisée en trois secteurs forestiers et est protégée depuis 1946 par l'arrêté de classement $\mathrm{N}^{\circ} 05574 / \mathrm{SE} / \mathrm{F}$ du 24/12/46. Le noyau central de la FCL jouit d'une protection intégrale et seule la recherche scientifique s'y déroule en abordant divers aspects liés à la végétation et à la faune. Elle est entourée de quinze villages qui sont réunis au sein du Comité de Gestion Participative de la Forêt (COGEPAF) et couvre une superficie totale estimée à 16250 ha dont 4777 ha sont couverts par le noyau central abritant la végétation naturelle de cette réserve forestière (Agbahoungba et al., 2016).

L'étude a été menée dans huit villages situés dans un rayon de $10 \mathrm{~km}$ autour du noyau de la forêt. Il s'agissait des villages comme Zalimey, Massi-Centre, Hlagba-Lonmè, Agadjaligbo, Donzoutoucoudja, Koto-Aïvèdji,
Akpè et Agbaga. Ils ont été choisis au hasard sur la liste des villages abritant les plantations forestières dans une bande de $10 \mathrm{~km}$ délimitée autour du Noyau central (Figure 1). Les villages tels que Zalimey, Agbaga et Agadjaligbo sont jouxtant au noyau central et se trouvent dans le périmètre forestier tandis que les cinq autres villages (Massi-centre, Hlagba-Lonmè, Donzoutoucoudja, KotoAïvèdji et Akpè) abritent uniquement des plantations forestières et sont situés en dehors du périmètre forestier. Ces huit villages sont situés dans les départements de l'Atlantique (Agbaga et Akpè) et du Zou (Zalimey, MassiCentre, Hlagba-Lonmè, Agadjaligbo, Donzoutoucoudja, Koto-Aïvèdji) (INSAE, 2016). Le milieu est caractérisé par un climat tropical de type subéquatorial avec une pluviométrie moyenne annuelle de $1100 \mathrm{~mm}$. Les sols dominants sont les vertisols et les sols sablo-argileux (Sinsin et al., 2003). Les groupes ethniques dominants sont : Holli, Fon, Aïzo. On y rencontre également d'autres groupes ethniques tels que les Adja, les Mina, les Yorubas provenant des départements voisins et du Togo.

\section{Collecte des données}

Nous avons adopté dans le cadre de cette recherche la définition selon Biaou et al. (2016) qu'un système agroforestier traditionnel est un mode d'utilisation des terres qui associe au moins un ligneux (arbres, arbustes, lianes, bambou, raphia, palmier, rôniers, etc.) à une culture annuelle (vivrière ou de rente) dans les espaces agraires (champ, jardin de case, plantation privée, jeune jachère) ayant une superficie d'au moins $500 \mathrm{~m}^{2}$ pour les (champs, jachères et plantations privées) et de superficies variables pour les jardins de case. Les caractéristiques floristiques de la zone étudiée ont permis de différencier quatre (4) types de systèmes agroforestiers traditionnels : champs, jardins de case, jachères et plantations privées. Dans cette étude, nous avions désigné par champs les systèmes de production dans lesquels les espèces ligneuses conservées constituent un support de production agricole pour les populations rurales mais aussi une 
source additionnelle d'aliments et de revenus (Badiane et al., 2019). Les jardins de case représentent un système agroforestier autour des maisons, ils fournissent une multiplicité de services et répondent à la fois aux objectifs alimentaires et économiques pour les agriculteurs, leur assurant une plus grande résilience dans de nombreuses régions intertropicales (Sardou et al., 2014). Les jachères représentent les zones de production agricole laissées au repos après plusieurs années de culture continue (Vodouhê et al., 2011). Enfin, les plantations privées sont des forêts plantées et régénérées artificiellement avec des espèces qui peuvent ne pas être locales, mais qui sont installées en monoculture afin de produire du bois et des produits dérivés de manière intensive en association avec les cultures vivrières (Triplet, 2016).

Dans chacun des villages choisis dans la zone d'étude, 30 placeaux carrés de $30 \mathrm{~m}$ x 30 $\mathrm{m}\left(900 \mathrm{~m}^{2}\right)$ ont été installés au hasard par système agroforestier. Cependant, en ce qui concerne les jardins de case toute la superficie de chacun des jardins a été prise en compte. Au total, 770 placeaux ont été inventoriés, dont 240 dans les champs, 240 dans les jardins de case, 240 dans les jachères et 50 dans les plantations privées. La plantation privée n'était pas une pratique très répandue dans la zone d'étude, ce qui a réduit considérablement le nombre de placeaux dans ce système agroforestier.

Les coordonnées géographiques du centre des placeaux d'inventaires forestiers ont été enregistrées à l'aide d'un récepteur GPS (Global Positioning System) à des fins d'analyses cartographiques. L'inventaire forestier a consisté en la prise des mesures dendrométriques tels que le diamètre sur les individus ayant un diamètre supérieur ou égal à $5 \mathrm{~cm}$ à $1,30 \mathrm{~m}$ du sol. Les signes d'exploitations éventuelles (émondage, écorçage, ébranchages et prélèvement des racines) ont été notés suivant une approche tactile (Rabiou et al., 2017) sur chaque espèce ligneuse alimentaire présente dans ces placeaux. L'identification des espèces s'est faite in-situ en utilisant des catalogues de plantes (de Souza et al., 2008) et la flore analytique du Bénin (Akoègninou et al., 2006). De plus, des herbiers de toutes les espèces ligneuses alimentaires trouvées dans les placeaux ont été réalisés pour l'identification ou la confirmation à l'Herbier National du Bénin.

\section{Analyse des données \\ Évaluation de la diversité des espèces ligneuses alimentaires}

La diversité des espèces ligneuses alimentaires a été évaluée par le biais de quelques indices de diversité tels que la richesse spécifique (S), l'indice de diversité de Shannon (H), l'équitabilité de Piélou (E) et l'indice de dominance de Simpson (D). La richesse spécifique (S) est le nombre total d'espèces inventoriées dans un système écologique.

L'indice de diversité de Shannon $(\mathrm{H})$ a été obtenu à partir de la formule suivante :

$H=-\sum_{i=1}^{s}\left(\frac{n_{i}}{n}\right) \log _{2}\left(\frac{n_{i}}{n}\right)$

$n_{i}$ est le nombre d'individus de l'espèce $\mathrm{i}, \mathrm{n}$ est le nombre d'individus de toutes les espèces inventoriées dans le placeau. L'indice de diversité de Shannon $H$ varie généralement de 1 à 5 bits. Si $H €[0 ; 2,5]$ alors $H$ peut être supposé faible ; Si $H$ est compris entre 2,6 et 3,9 alors il peut être supposé moyen ; Si $H$ est compris entre 4 et 5 alors il peut être supposé élevé (Mbaiyetom et al., 2021).

L'équitabilité de Piélou $\left(E_{q}\right)$ mesure le degré de diversité des écosystèmes par rapport au maximum possible. Sa formule se présente comme suit :

$$
\begin{aligned}
& E_{q}=\frac{H}{H_{\max }} \text { avec } H_{\text {max }} \\
& =\log _{2} S
\end{aligned}
$$

$H_{\text {max }}$ est la valeur maximale de l'indice de diversité de Shannon du système agroforestier, $\mathrm{S}$ est le la richesse spécifique et $H$ l'indice de diversité de Shannon. $\mathrm{E} q$ a une valeur comprise entre 0 et 1 . Elle est faible 
lorsque $\mathrm{E} q$ est inférieure à 0,6 ; moyenne si $\mathrm{E} q$ est compris entre 0,6 et 0,7 puis élevée si $\mathrm{E} q$ est supérieure ou égal à 0,8 . Elle est très utile pour comparer les dominances potentielles entre les systèmes.

L'indice de diversité de Simpson (D) permet de mieux évaluer la diversité et est basé sur la présence et l'abondance des espèces. Il indique la diversité maximale quand sa valeur est proche de 0 et la diversité minimale quand sa valeur est proche de 1 (Zerbo et al., 2014). Il a été calculé par la formule suivante :

$$
D=\frac{1}{n(n-1)} \sum_{i=1}^{s} n_{i}\left(n_{i}\right.
$$

$n_{i}$ est le nombre d'individus de l'espèce i dans le système agroforestier considéré et $\mathrm{n}$ est le nombre total d'individus de toutes les espèces dans le système agroforestier.

L'indice de similarité de Jaccard et le diagramme de Venn ont été utilisés pour évaluer la similitude floristique entre les systèmes agroforestiers. L'indice de Jaccard a été utilisé pour évaluer la similitude floristique entre deux systèmes (Ndong et al., 2015). Le diagramme de Venn a permis la représentation graphique d'opérations telle que l'intersection des espèces ligneuses alimentaires effectuées sur l'ensemble des quatre systèmes agroforestiers traditionnels. L'indice de similarité de Jaccard représente la diversité $\beta$ qui est le taux de remplacement des espèces et individus lors $\mathrm{du}$ passage d'un système agroforestier à un autre. Il a pour but de caractériser objectivement et quantitativement le degré de ressemblance de deux systèmes. L'indice de Jaccard a été obtenu à partir de l'équation 4.

$$
\text { Jaccard (systèmes A et B) }=\frac{c}{a+b-c}
$$

Avec a et $b=$ le nombre d'espèces présentes dans les systèmes $\mathrm{A}$ et $\mathrm{B}$ respectivement, $\mathrm{c}=\mathrm{le}$ nombre d'espèces communes aux deux systèmes A et B. La valeur de l'indice est égale à 0 lorsque les deux systèmes ne présentent aucune similarité. Par contre, elle est égale à 1 , si les deux systèmes sont totalement identiques.

\section{Évaluation des caractéristiques dendrométriques des espèces ligneuses alimentaires \\ Calcul de la variation des indices de valeur d'importance}

L'IVI caractérise la place qu'occupe chaque espèce par rapport à l'ensemble des espèces dans les écosystèmes. Il est utilisé pour évaluer la prépondérance spécifique en forêts tropicales selon la formule (Pereki et al., 2013) :

IVI = Dominance relative + Densité relative + Fréquence relative

où :

- la dominance relative d'une espèce est le rapport entre sa surface terrière sur l'ensemble des placeaux réalisées et la surface terrière de toutes les espèces (toutes espèces confondues) ;

- la densité relative d'une espèce est le rapport entre sa densité absolue et la densité totale absolue de toutes les espèces ;

- la fréquence relative d'une espèce est le produit par 100 du rapport entre sa fréquence spécifique et le total des fréquences spécifiques de toutes les espèces.

Afin de faire la caractérisation structurale des systèmes agroforestiers, les paramètres dendrométriques (densité, diamètre moyen et quotient entre classes consécutives) ont été calculés et la distribution en classe de diamètre a été établie. Les caractéristiques dendrométriques ont été évaluées pour les 5 premières espèces ayant la plus forte valeur de l'IVI par système agroforestier.

Densité et diamètre moyen

La densité est le nombre moyen d'arbres par unité de surface, elle s'exprime en individus / ha et est donnée par la formule.

$N=\frac{n}{S}$

Avec $\mathrm{n}$ le nombre d'individus dans le placeau et $\mathrm{S}$ la surface du placeau exprimée en hectare. Diamètre moyen $(\mathrm{Dg})$

Il est le diamètre de l'arbre ayant une surface terrière égale à la surface terrière moyenne du système agroforestier considéré. Il est exprimé en $\mathrm{cm}$. 
$D_{g}=\sqrt{\frac{1}{n} \sum_{1}^{n} d_{i}^{2}}$

Avec $d_{i}$ le diamètre (en $\mathrm{cm}$ ) de l'individu i et $\mathrm{n}$ le nombre d'individus de l'espèce $i$ dans le placeau.

Ces paramètres calculés par placeau ont été soumis au modèle linéaire généralisé en prenant comme facteur les systèmes agroforestiers.

Calcul du quotient entre classes consécutives

Afin d'apprécier la stabilité des peuplements de chacune des espèces ligneuses alimentaires, les quotients entre deux classes de diamètre consécutives ont été utilisés en se basant sur la formule suivante :

$$
Q=\frac{N_{(i-1)}}{N_{i}}
$$

Avec $N_{i}$ l'effectif de la classe i et $N_{(i-1)}$ l'effectif de la classe précédente.

Les quotients ont été ensuite représentés suivant les classes de diamètre. Les quotients fluctuant entre les classes successives indiquent une population instable et des quotients constants expriment une population stable. Cette méthode de distribution de la structure en diamètre des espèces ligneuses a été déjà utilisée par d'autres auteurs dans des études similaires (Assogba et al., 2020). La structure en diamètre des espèces a été analysée au moyen de la répartition des quotients entre deux classes de diamètre consécutives des individus ligneux et a été représentée par des histogrammes.

Les données collectées sur la pression anthropique au niveau des espèces ligneuses ont été analysées à l'aide des outils de la statistique descriptive tels que la fréquence. Enfin, les résultats de l'évaluation des menaces sur les individus des espèces ligneuses alimentaires dans les systèmes agroforestiers ont été présentés sous formes d'histogrammes.

Le logiciel R-3.6.1 a été utilisé pour traiter et analyser les données.

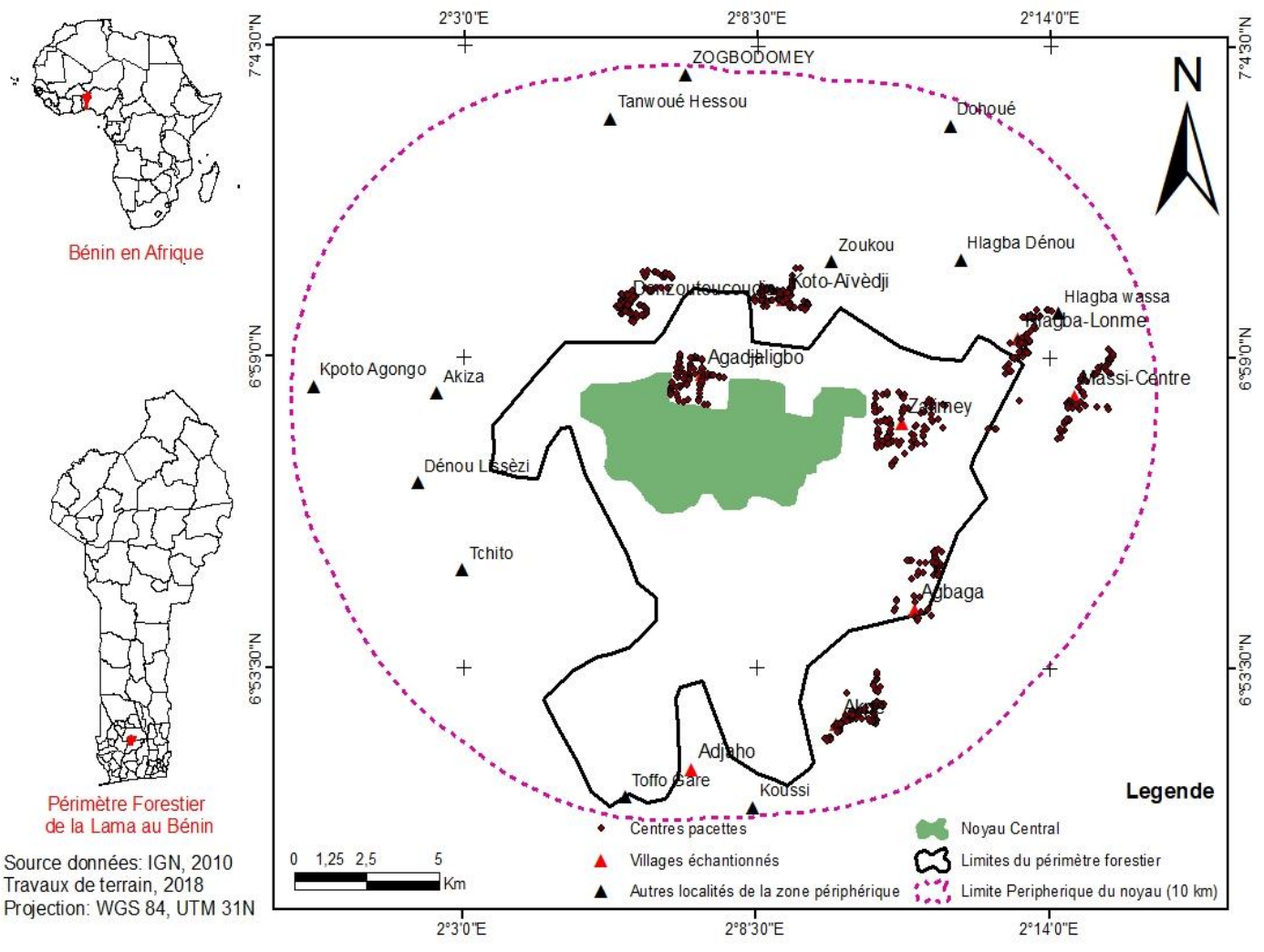

Figure 1 : Carte du milieu d'étude. 


\section{RESULTATS}

\section{Diversité des espèces ligneuses alimentaires dans les systèmes agroforestiers}

Quarante-quatre (44) espèces végétales réparties dans 29 familles ont été identifiées dans les systèmes agroforestiers de la zone périphérique du noyau central de la forêt classée de la Lama (Tableau 1). Quarante et une (41) espèces ont été recensées respectivement dans les champs et les jardins de case, quarante-quatre (44) dans les jachères et vingt-huit (28) dans les plantations privées. En termes du nombre d'individus, Spondias mombin (114 individus) était l'espèce végétale la plus abondante dans les jardins de case et Adansonia digitata (70 individus) dans les champs. Les familles les plus représentées étaient: les Leguminosae (8 espèces) et les Moraceae (4 espèces).

La richesse spécifique des espèces ligneuses alimentaires était la plus élevée dans les jachères (44 espèces) par rapport aux autres systèmes agroforestiers. La plus faible richesse en espèces a été observée dans les plantations privées (28 espèces). Les jardins de case et les champs ont enregistré chacun 41 espèces. La valeur la plus élevée de l'indice de diversité de Shannon $(1,44)$ a été enregistrée dans les jachères tandis que la valeur la plus élevée de l'équitabilité de Piélou $(0,93)$ a été enregistrée dans les plantations privées (Tableau 2).

La valeur la plus élevée de l'indice de dominance de Simpson $(D=0,08)$ a été enregistrée au niveau des jardins de case tandis que la valeur la plus faible $(\mathrm{D}=0,04)$ a été enregistrée dans les plantations privées. Ainsi, la probabilité de rencontrer des espèces ligneuses alimentaires dans les jardins de case est supérieure à celle de les rencontrer dans les plantations privées (Tableau 2).

\section{Similitudes entre les systèmes agroforestiers}

La composition floristique ne différait pas d'un système à un autre car dans tous les cas considérés, l'indice de similarité dépasse 0,5 (Tableau 3). On note cependant la ressemblance la plus élevée entre les jachères et les jardins de case $(0,86)$. Le nombre important d'espèces communes (41) entre ces deux systèmes explique cette ressemblance. $\mathrm{La}$ valeur la plus faible de cet indice a été enregistrée entre les jachères et les plantations privées $(0,55)$.

Il a été noté que 28 espèces étaient communes aux quatre systèmes agroforestiers (Figure 2). Les champs et plantations privées avaient 27 espèces en commun. Quarante-un (41) espèces étaient communes aux jachères et champs. Vingt-huit (28) espèces étaient communes aux plantations privées et jardins de case. Trente-huit (38) espèces étaient communes aux champs et aux jardins de case. Quarante et une (41) espèces étaient communes aux jachères et aux jardins de case.

\section{Importance écologique des espèces ligneuses alimentaires}

La caractérisation de la végétation a permis d'évaluer l'importance des espèces ligneuses alimentaires en se basant sur l'Indice de la Valeur d'Importance (IVI).

Dans les jachères, l'indice IVI a varié de $0,80($ A. digitata $)$ à $0,10($ J. curcas, $N$. laevis et $B$. sapida) (Figure 3 a). Dans les champs, $A$. digitata a également enregistré l'IVI le plus élevé 1,01 tandis que $S$. latifolius $(0,19)$ a obtenu la plus faible valeur (Figure $3 \mathrm{~b}$ ). Dans les jardins de case, $S$. mombin a présenté l'IVI le plus élevé $(0,55)$ tandis que $J$. gossypiifolia, $S$. siamea et $A$. digitata ont enregistré les valeurs IVI les plus faibles (Figure $3 \mathrm{c}$ ). Dans les plantations privées, A. digitata $(0,8)$ a présenté également l'IVI le plus élevé (Figure $3 \mathrm{~d})$.

\section{Caractéristiques dendrométriques des espèces ligneuses alimentaires}

La densité moyenne la plus élevée des espèces ligneuses alimentaires a été observée dans les jardins de case $(29,12$ arbres / ha) et les densités les plus faibles dans les champs (21,71 arbres / ha). Les gros arbres ( $B$. aethiopum et $A$. digitata) de diamètre compris entre 79,06 cm et $118,24 \mathrm{~cm}$ étaient les plus répandues dans les champs, jachères et plantations privées. Dans les jardins de case, les arbres de diamètre $9,79 \mathrm{~cm}$ et $29,50 \mathrm{~cm}$ étaient abondants (Tableau 4). 


\section{Structure en diamètre des espèces}

La Figure 4 illustre les quotients calculés pour les classes de diamètre consécutives. Les champs, les plantations privées et les jachères révèlent une faible présence de jeunes individus contre une forte présence des individus à gros diamètre [110130[. Parmi tous les systèmes agroforestiers étudiés, les jardins de case étaient des systèmes agroforestiers plus riches en individus de petit diamètre.

Actions délétères causées par les populations locales sur les espèces ligneuses alimentaires dans les systèmes agroforestiers

Les individus des espèces ligneuses alimentaires sont exposés à diverses actions néfastes causées par l'homme notamment l'ébranchage, l'écorçage du tronc d'arbre, l'émondage et le prélèvement des racines dans tous les systèmes agroforestiers. Le taux d'ébranchage était le plus élevé dans les jardins de case (48\%). La plus faible proportion (32\%) d'individus ébranchés a été enregistrée dans les jachères. L'écorçage variait selon les systèmes agroforestiers. Plus de $40 \%$ de plantes ligneuses alimentaires des systèmes agroforestiers dans la zone périphérique du noyau central de la forêt classée de la Lama étaient émondées dans les jardins de case. Les taux les plus faibles (22\%) d'individus émondés ont été observés dans les jachères. Le prélèvement des racines était plus accentué dans les champs $(10 \%)$ et plus faible $(4 \%)$ dans les plantations privées (Figure 5).

Tableau 1 : Liste exhaustive des espèces ligneuses alimentaires répertoriées et le nombre d'individus par système agroforestier traditionnel.

\begin{tabular}{|c|c|c|c|c|c|c|}
\hline \multirow[b]{2}{*}{ Espèces } & \multicolumn{5}{|c|}{ Nombre d'individus par systèmes agroforestiers } & \multirow[b]{2}{*}{ Familles } \\
\hline & $\begin{array}{l}\text { Jardins } \\
\text { de case }\end{array}$ & Champs & Jachères & $\begin{array}{l}\text { Plantations } \\
\text { privées }\end{array}$ & Total & \\
\hline 1) Adansonia & & & & & & \\
\hline $\begin{array}{l}\text { digitata } \mathrm{L} . \\
\text { 2) Antiaris toxicaria }\end{array}$ & 5 & 70 & 42 & 11 & 128 & Malvaceae \\
\hline Lesch. & 3 & 2 & 3 & 2 & 10 & Moraceae \\
\hline $\begin{array}{l}\text { 3) Anogeissus } \\
\text { leiocarpa (Dc.) }\end{array}$ & & & & & & \\
\hline Guill. \&Perr., & 2 & 34 & 14 & 4 & 54 & Combretaceae \\
\hline 4) Bombax sp & 32 & 6 & 15 & 2 & 55 & Malvaceae \\
\hline 5) Annona & 1 & 13 & 33 & 3 & 50 & Annonaceae \\
\hline 6) Blighia sapida & 1 & 13 & & & & Ammonaceae \\
\hline Konig & 9 & 8 & 17 & 3 & 37 & Sapindaceae \\
\hline 7) Borassus & & & & & & \\
\hline $\begin{array}{l}\text { aethiopum Mart., } \\
\text { 8) Crateva }\end{array}$ & 3 & 31 & 14 & 4 & 52 & Arecaceae \\
\hline $\begin{array}{l}\text { adansonii DC. } \\
\text { 9) Croton }\end{array}$ & 15 & 1 & 3 & 0 & 19 & Capparaceae \\
\hline $\begin{array}{l}\text { gratissimus Bureh., } \\
\text { 10) Chrysophyllum }\end{array}$ & 44 & 2 & 8 & 1 & 55 & Euphorbiaceae \\
\hline albidum G.Don, & 1 & 0 & 1 & 0 & 2 & Sapotaceae \\
\hline
\end{tabular}


11) Cola nitida

Sebott \& EndI.,

12) Dialium

guineense WiIld.,

13 Dichapetalum

madagascariense

Poir.,

2

1

1

0

4 Malvaceae

14) Diospyros

mespiliformis

Hochst.

1

15) Eucalyptus

camaldulensis

Dehn.,

2

16) Erythrina

senegalensis DC.,

29

17) Ficus

exasperataVahl,

18) Ficus sur

Forssk.,

19) Gardenia

ternifolia

Sehumaeh.

\&Thonn.

1

20) Holarrhena.

floribunda G. Don

22) Jatropha curcas

L.,

23) Jatropha

gossypiifolia L.,

24) Khaya

senegalensis A.

Juss.,

10

25) Kigelia africana

Benth.,

2

26) Lecaniodiscus

cupanioides Planch.

27) Milicia excelsa

C. C. Berg,

28) Morinda lucida

Benth.,

29) Moringa

oleifera Lam.,

30) Newbouldia

laevis (P. Beauv.)

33

5

8

33

31) Parkia

biglobosa (Jacq.) R.

Br. ex Benth.,

2

32) Phoenix

reclinata Jacq.,

1

0

2

3

3

9 Ebenaceae

4

39

31

5

14

15

0

2 Dichapetalaceae

4 Leguminosae

5

1

0

3 Rubiaceae

8

15

0

29 Apocynaceae

14 Irvingiaceae

38 Euphorbiaceae

3

1

$13 \quad 13$

3

39 Meliaceae

8 Bignoniaceae

2

4

3

13

3

25

18

19

20

7

3
3
48 Leguminosae

$7 \quad$ Sapindaceae

24 Moraceae

34 Rubiaceae

17 Moringaceae

80 Bignoniaceae

6 Arecaceae 
33) Pterocarpus

santalinoides L'Hér.

Ex Dc.,
34) Sarcocephalus

19

5

13

2

39 Leguminosae

latifolius (Sm.) E.
A. Bruce,
35) Oxytenanthera abyssinica

3

20

15

3

41 Rubiaceae

(A.Rich.)

0

4

7

11 Poaceae

36) Senna alata (L.)

Roxb.,

2

3

7 Leguminosae

37) Senna siamea

(Lam.)

16

$$
11
$$

9

43 Leguminosae

38) Spondias

mombin L.,

40

55

212 Anacardiaceae

39) Synsepalum

dulcificum

(Schumach.

\&Thonn.)

40) Tamarindus

indica $\mathrm{L}$.,

0

$1 \quad 0$

2 Sapotaceae

41) Tetrapleura

tetraptera

(Schumach.

\&Thonn.)

42) Uvaria chamae

P. Beauv.,

2

1

2

0

5 Leguminosae

43) Vitex doniana

Sweet,

1

1

0

3 Annonaceae

44) Zanthoxylum

10

28

30

7

75 Lamiaceae

zanthoxyloides

(Lam.)

2

6

5

14 Rutaceae

Tableau 2: Diversité des espèces ligneuses alimentaires dans les systèmes agroforestiers traditionnels.

\begin{tabular}{lcrcc}
\hline Paramètres & Jachère & \multicolumn{1}{c}{ Champs } & Jardin de case & Plantation privée \\
\hline Richesse (S) & 44 & 41 & 41 & 28 \\
Shannon (H) & 1,44 & 1,34 & 1,23 & 1,35 \\
Piélou (E) & 0,88 & 0,83 & 0,77 & 0,93 \\
Simpson (D) & 0,05 & 0,06 & 0,08 & 0,04 \\
\hline
\end{tabular}


Tableau 3 : Similarité entre les systèmes agroforestiers traditionnels.

\begin{tabular}{lcccc}
\hline $\begin{array}{l}\text { Systèmes agro- } \\
\text { forestiers }\end{array}$ & Jardin de case & Champs & Jachères & Plantations privées \\
\hline Jardins de case & 1 & - & - & - \\
Champs & 0,71 & 1 & - & - \\
Jachères & 0,86 & 0,77 & 1 & - \\
Plantations privées & 0,63 & 0,66 & 0,55 & 1 \\
\hline
\end{tabular}

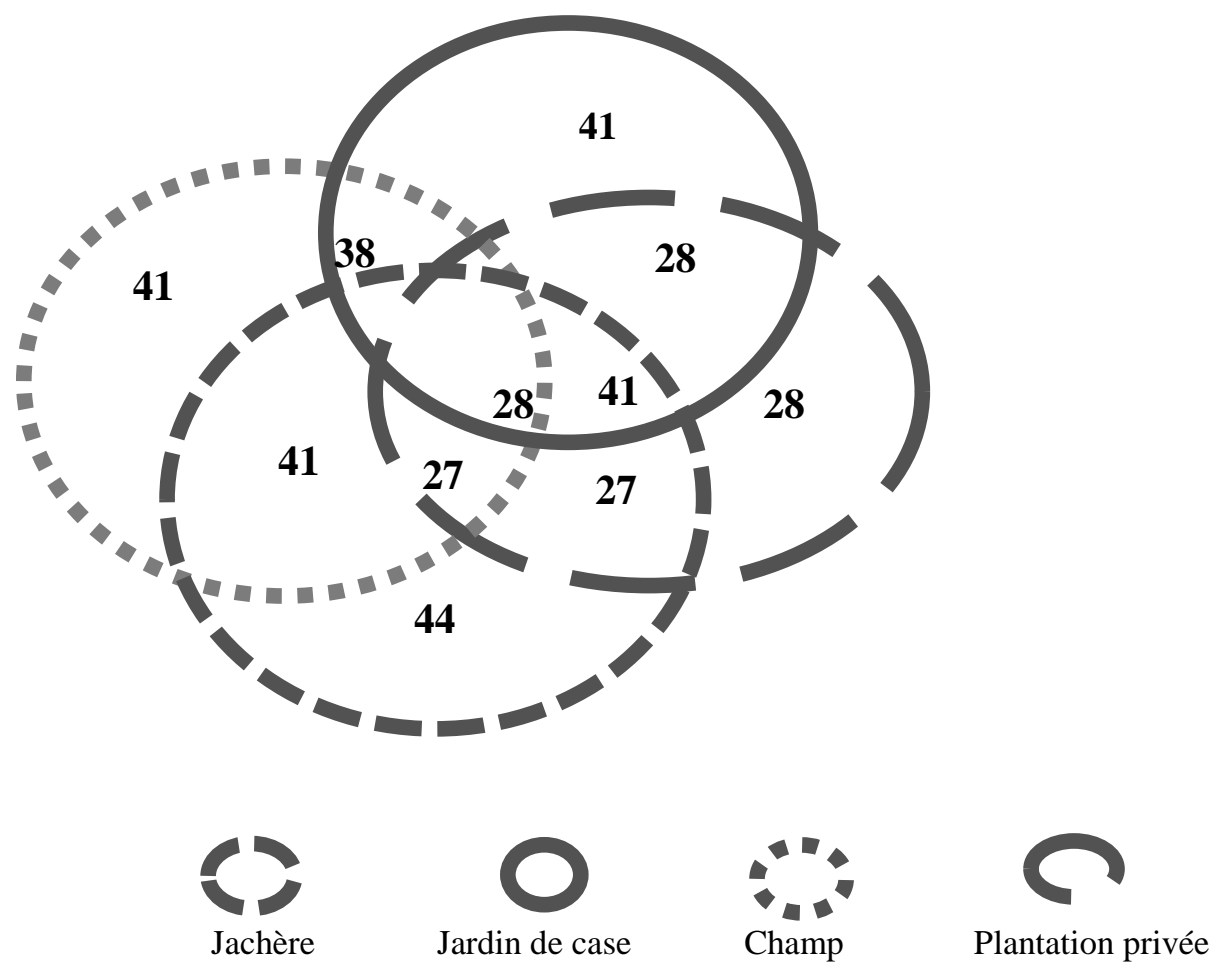

Figure 2 : Diagramme de Venn montrant la similarité entre les systèmes. 

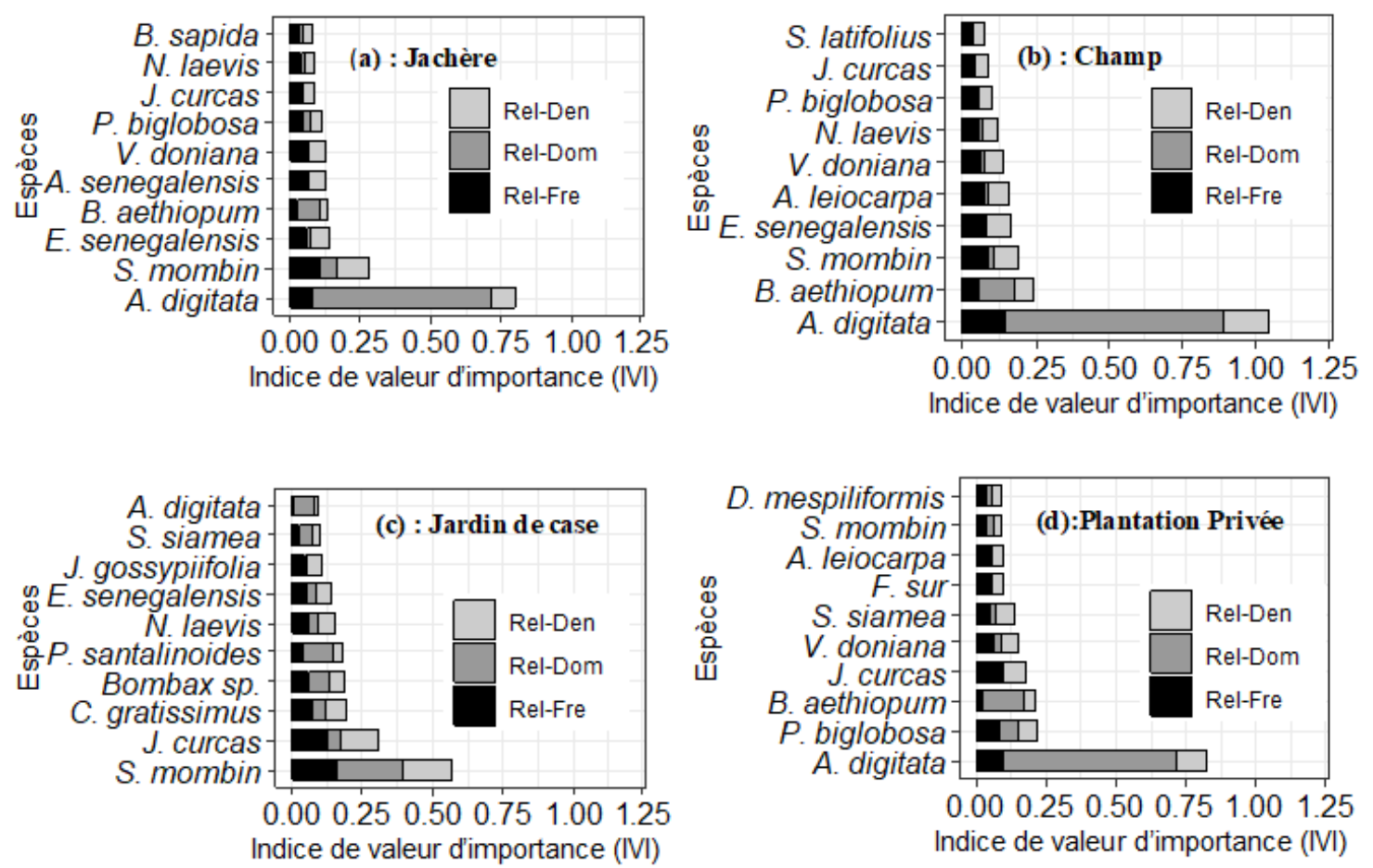

Rel-Den $=$ Densité Relative $;$ Rel-Dom $=$ Dominance Relative $;$ Rel-Fre $=$ Fréquence Relative

Figure 3 : Variation des indices de valeur d'importance des espèces ligneuses alimentaires par système agroforestier.

Tableau 4 : Densité et diamètre moyen des espèces ligneuses importantes des quatre systèmes agroforestiers traditionnels.

\begin{tabular}{|c|c|c|c|c|}
\hline Espèces & $\begin{array}{c}\begin{array}{c}\text { Densité } \\
\text { (arbres /ha) }\end{array} \\
\end{array}$ & cv $(\%)$ & $\begin{array}{l}\text { Diamètre } \\
\text { Moyen (cm) }\end{array}$ & $\operatorname{cv}(\%)$ \\
\hline \multicolumn{5}{|c|}{ Jachère } \\
\hline A. digitata & 13,73 & 44,82 & 100,86 & 42,88 \\
\hline S. mombin & 14,22 & 42,31 & 7,97 & 33,22 \\
\hline E. senegalensis & 15,56 & 49,94 & 63,73 & 22,02 \\
\hline B. aethiopum & 13,25 & 33,71 & 16,86 & 50,77 \\
\hline A. senegalensis & 13,95 & 67,51 & 24,05 & 69,39 \\
\hline Global & 22,92 & 56,46 & 31,90 & 96,09 \\
\hline \multicolumn{5}{|c|}{ Champ } \\
\hline A. digitata & 13,41 & 40,35 & 118,24 & 10,13 \\
\hline B. aethiopum & 15,66 & 56,52 & 64,78 & 31,06 \\
\hline S. mombin & 13,07 & 32,89 & 19,3 & 84,08 \\
\hline E. senegalensis & 14,56 & 46,08 & 16,12 & 57,52 \\
\hline A. leiocarpa & 13,03 & 32,79 & 19,08 & 61,94 \\
\hline Global & 21,71 & 57,57 & 46,35 & 90,28 \\
\hline
\end{tabular}




\section{Jardins de case}

$\begin{array}{lcccr}\text { S. mombin } & 14,58 & 43,02 & 18,21 & 58 \\ \text { J. curcas } & 14,06 & 45,05 & 9,79 & 43,59 \\ \text { C. } \text { gratissimus } & 12,87 & 37,71 & 13,17 & 52,04 \\ \text { Bombax sp. } & 11,85 & 34,23 & 18,41 & 68,5 \\ \text { P.santalinoides } & 11,11 & 0,00 & 29,5 & 73,12 \\ \text { Global } & 29,12 & 44,48 & 18,66 & 68,89\end{array}$

\section{Plantations privées}

\begin{tabular}{lrrrr} 
A. digitata & 15,28 & 37,64 & 97,7 & 37,2 \\
P. biglobosa & 11,11 & 0,00 & 32,42 & 76,67 \\
B. aethiopum & 2,2 & 70,71 & 79,06 & 1,29 \\
J. curcas & 11,11 & 0,00 & 6,60 & 30,42 \\
V. doniana & 13,33 & 37,27 & 24,58 & 74,01 \\
Global & 22,22 & 59,76 & 35,24 & 100,58 \\
\hline
\end{tabular}

CV : Coefficient de variation.
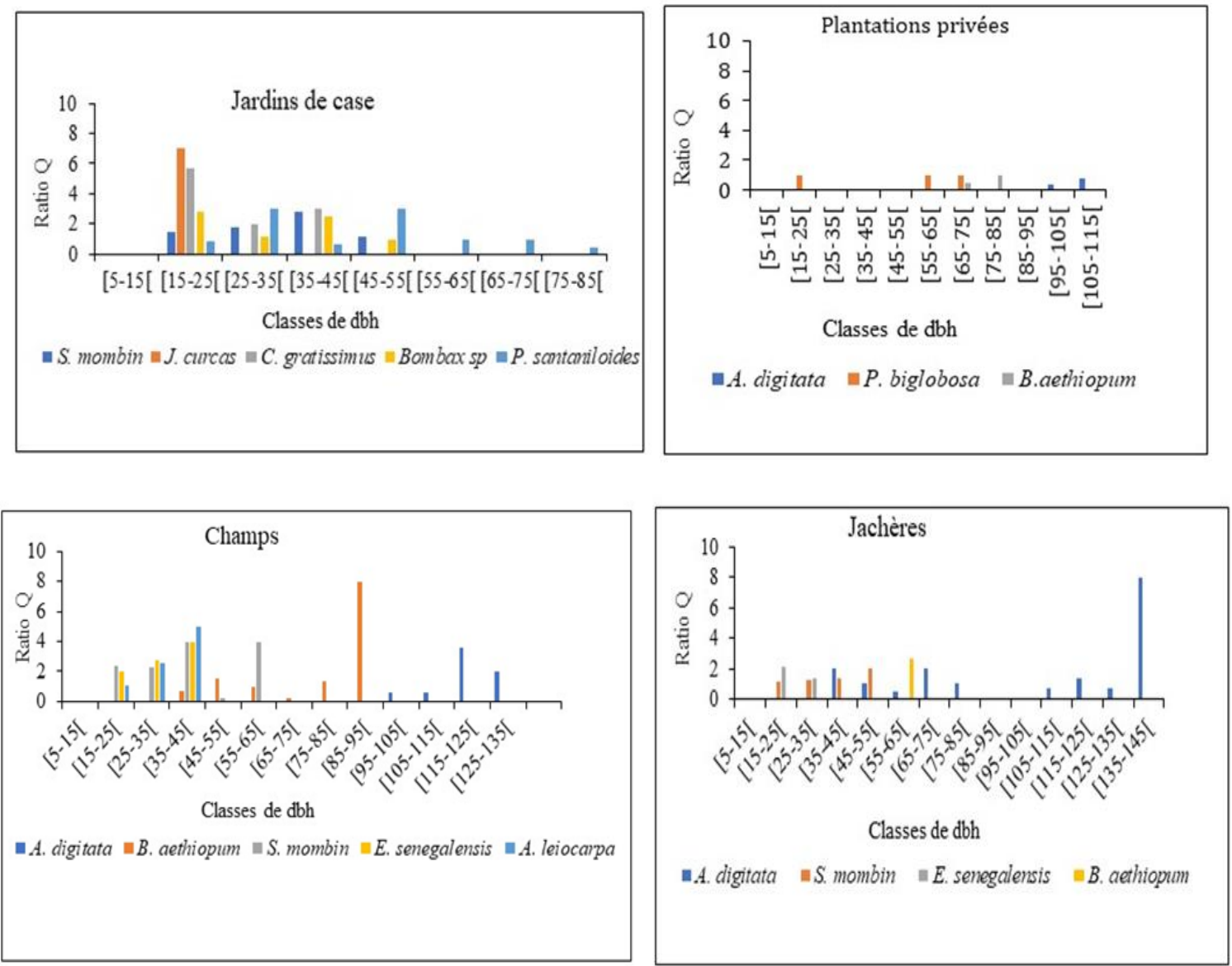

Figure 4 : Quotient des classes consécutives. 


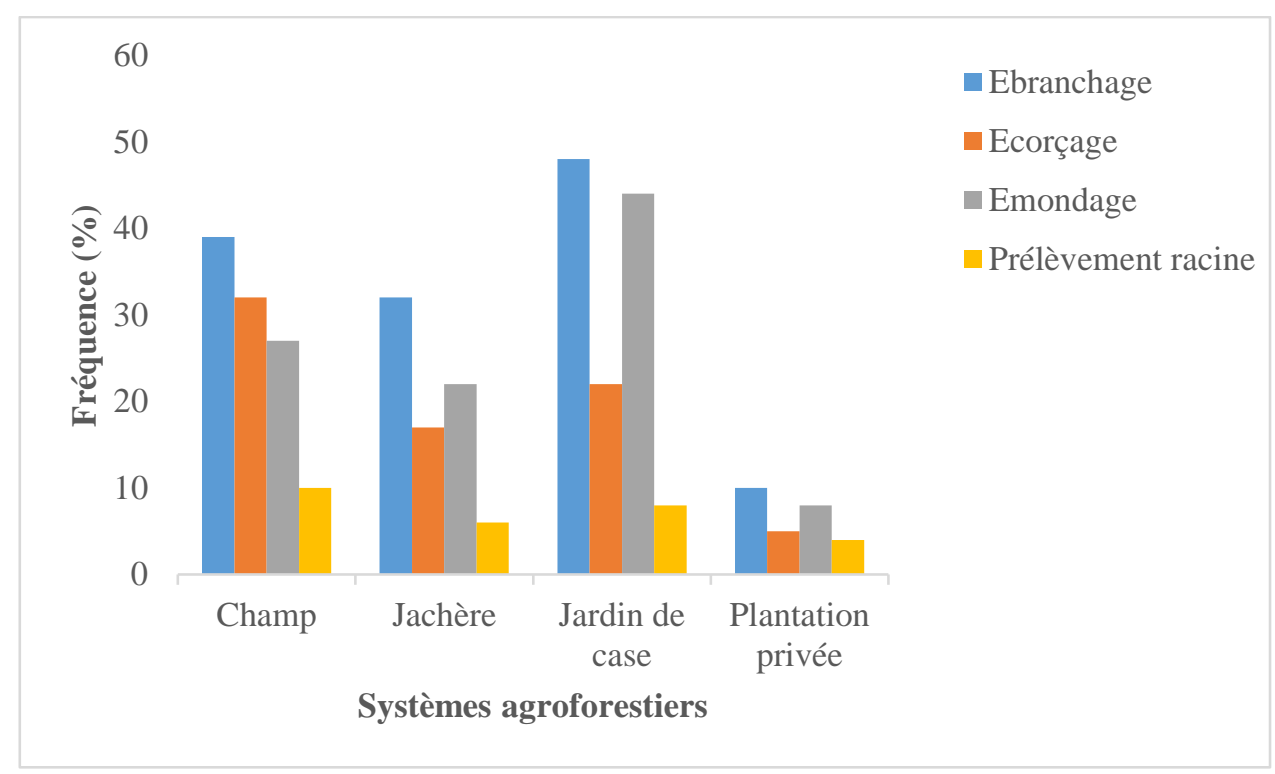

Figure 5 : Pourcentage des actions délétères par les populations sur les espèces ligneuses alimentaires dans les systèmes agroforestiers.

\section{DISCUSSION}

Les jardins de case, véritables sanctuaires pour la conservation des plantes ligneuses alimentaires

La diversité et la structure de quatre systèmes agroforestiers (jardins de case, champs, jachères et plantations privées) dans la périphérie du noyau central de la forêt classée de la Lama (FCL) ont été évaluées. La densité moyenne des espèces ligneuses alimentaires dans les systèmes agroforestiers était la plus élevée dans les jardins de case $(29,12$ individus/ha) et la plus faible dans les champs (21,71 individus/ha). Les plantes étaient plus conservées dans les jardins de case que dans les champs. Ce qui témoigne de la contribution des jardins de case à la conservation de la biodiversité au Bénin comme l'ont illustré plusieurs travaux (Salako et al., 2014 ; Idohou et al., 2014). De même, ce résultat concorde avec les travaux de Sardou et al. (2014) en Haïti qui stipulent que les jardins de case constituent des réservoirs de la diversité biologique. En effet, pour des raisons alimentaires et médicinales les populations conservent et/ou cultivent des plantes à côté de leur concession (Idohou et al., 2014 ; Gbedomon et al., 2016).
C'est le cas par exemple de S. mombin, $C$. gratissimus, J. curcas et de E. senegalensis.

Parmi les espèces ligneuses de grande importance, A. digitata et $S$. mombin étaient les plus abondantes. Ambé (2001) a rapporté que S. mombin fait partie des 55 espèces ligneuses les plus utilisées à Séguéla (Côte d'Ivoire) en raison de son importance essentiellement alimentaire et fourragère, ce qui entraîne une fréquence élevée des arbres dans les jardins de case. Cette étude a montré la présence de quelques individus de $A$. digitata dans les jardins de case et seuls quelques arbres isolés étaient conservés dans les champs, les jachères et les plantations privées. Avana-Tientcheu et al. (2019) au Tchad ont obtenu des résultats similaires sur $A$. digitata dans les jachères, champs et jardins de case.

Par ailleurs, ces résultats témoignent de la volonté des populations de conserver cette espèce ainsi que d'autres espèces qui leur sont utiles. C'est le cas de A. leiocarpa et $B$. aethiopum conservés dans les jardins de case au Nigéria selon les travaux de Odebiyi et al. (2004). 


\section{Structure diamétrique des espèces ligneuses alimentaires}

La distribution par classe de diamètre est utilisée pour comprendre la dynamique des arbres et peut être utilisée pour évaluer l'impact de la pression anthropique sur la population des arbres (Badiane et al., 2019). L'analyse des paramètres dendrométriques a révélé la présence des individus de grands diamètres dans les champs. Par exemple, A. digitata occupe une classe de diamètre comprise entre [150-170[cm. Selon Mbaiyetom et al. (2021), les faibles densités des classes de gros arbres résultent de la sélection naturelle et sont en fait les semenciers qui assurent la pérennité du peuplement. La rareté des individus dans les classes intermédiaires indique que les plantules arrivent rarement à maturité. De même, l'absence de jeunes plants de $A$. digitata pourrait être liée à la consommation des bourgeons des jeunes plants par les populations locales et les animaux. Cela pourrait être également lié à la volonté des agriculteurs d'entretenir et de protéger les gros arbres soit pour leur rôle fertilisant, leur ombrage ou comme bois sacré d'une part et d'autre part en fonction de leurs valeurs économiques et nutritives. La protection améliore la structure des peuplements naturels comme observée par Assogbadjo et al. (2010) sur des peuplements de Anogeissus leiocarpa Guill, Perr. Abdourhamane et al. (2017) ont également rapporté ces observations sur la structure démographique de Sclerocarya birrea (A. Rich.) Hochst dans les champs au Niger. Koda et al. (2016) explique que la dominance des individus de gros diamètre de $A$. digitata observé dans les champs pourrait être liée aux facteurs climatiques conjugués aux facteurs anthropiques qui constituent les principaux facteurs de sélection des individus dans ce système. De même, la faible densité des individus adultes témoigne d'un environnement fortement perturbé avec une surexploitation des individus (Barmo et al., 2019).

Par contre, dans les plantations privées, l'absence d'individus dans les classes de diamètre $[25-35[\mathrm{~cm} ;[35-45[\mathrm{~cm}$ et $[85-95[\mathrm{~cm}$ pour les espèces indique une pression humaine sélective sur les individus de petit et grand diamètre dans ce système. L'inexistence des arbres dans ces classes de diamètre pourrait être la résultante d'une ancienne exploitation clandestine par la population. La pression anthropique (coupes sélectives, feux de végétation) serait donc à l'origine des perturbations affectant la régénération des espèces. Il est donc important pour l'utilisation durable des ressources de $P$. biglobosa et $V$. doniana de considérer l'enrichissement des plantations privées en ces espèces surtout dans les périphéries du noyau central de la FCL où la densité maximale ne dépasse pas 11 et 13 individus par hectare respectivement pour $P$. biglobosa et $V$. doniana.

Dans les jardins de case, la répartition des individus en classes de diamètre a révélé une diminution progressive du nombre d'individus lorsque la classe de diamètre augmente avec une prédominance des individus de petits diamètres et une rareté des individus à partir de la classe de diamètre [55$65[\mathrm{~cm}$. Selon Agbogan et al. (2015), les densités élevées des classes de faible diamètre assurent l'avenir de la formation naturelle et cela peut être considéré comme un indicateur de l'équilibre de ce système. Une telle distribution est typique des populations stables, susceptibles de se renouveler par la régénération naturelle (Barmo et al., 2019). Kebenzikato et al. (2014) ont observé de telles distributions pour la structure de A. digitata dans les jardins de case au Togo.

\section{Pressions anthropiques sur les espèces}

Dans les systèmes agroforestiers autour du noyau central de la FCL, l'écorçage des troncs et le prélèvement des racines sont les techniques de récoltes les plus utilisées avec respectivement des taux de $10 \%$ et $24 \%$. Ces deux techniques de récolte pourraient poser des problèmes quant à la durabilité des ressources végétales car ne garantissant pas toujours la survie des espèces (Balna et al., 2015). L'écorçage est souvent responsable de troubles physiologiques chez les arbres (Traore et al., 2011). L'écorçage excessif des espèces telles que $K$. senegalensis, $A$. digitata et $P$. biglobosa sont répandus dans la zone d'étude et 
compromettent la survie des espèces. Les racines de $P$. santaniloides, A. leiocarpa et $S$. siamea sont fréquemment collectées et utilisées à des fins médicinales dans la zone d'étude. La forte pression exercée sur les organes végétaux ne sert pas seulement à l'alimentation humaine et à la médecine, mais aussi à la construction de logements et à la nourriture des animaux ou du bétail. Cette situation pourrait constituer une inquiétude pour une meilleure conservation des espèces dans les systèmes agroforestiers de la FCL car les populations de ces régions tirent l'essentiel de leurs biens de cet écosystème (Djodjouwin et al., 2011). En l'état actuel, il serait important de définir un plan d'aménagement autour de ces espèces utiles pour les populations locales en vue de leur conservation durable.

\section{Conclusion}

L'objectif de cette étude était de contribuer à une meilleure compréhension de l'état actuel de la diversité des espèces ligneuses alimentaires autour du noyau central de la forêt classée de la Lama. Les enquêtes ainsi que les travaux de terrain ont montré que les champs sont caractérisés par un peuplement dominant d'individus de grand diamètre. L'espèce A. digitata a fait l'objet de soins particuliers et est conservée loin des activités humaines par certains résidents locaux pour la nourriture et les rituels. Par ailleurs, les populations devraient être régulièrement sensibilisées sur l'importance de préserver les espèces endogènes dont l'extinction affectera non seulement la biodiversité nationale mais aussi les valeurs socio-culturelles qui leur sont liées. Les stratégies des programmes de reboisement visant à intégrer les plantes alimentaires dans les systèmes agroforestiers pourraient contribuer à réduire la pression sur la réserve forestière de la Lama.

\section{CONFLIT D'INTERETS}

Les auteurs déclarent qu'il n'y a aucun conflit d'intérêts.

\section{CONTRIBUTIONS DES AUTEURS}

BEJA a contribué à la collecte des données, à l'analyse des données et à la rédaction du manuscrit. $\mathrm{AH}$ a fourni les informations de base sur la forêt de la Lama. Il a aussi travaillé de connivence avec BEJA dans le traitement des données écologiques, à l'analyse des données et à la correction du manuscrit. YSSB, GCA, EAP, YCS, AEA, RGK ont contribué à la lecture et la correction du présent manuscrit.

\section{REMERCIEMENTS}

Les auteurs remercient la Banque Mondiale pour le financement à travers le centre d'Excellence Africain sur les Changements Climatiques, la Biodiversité et l'Agriculture Durable (CEA-CCBAD) en Côte d'Ivoire et les évaluateurs pour avoir contribué à l'amélioration de la qualité scientifique de cet article.

\section{REFERENCES}

Abdourhamane H, Rabiou H, Diouf A, Morou B, Mahamane A, Bellefontaine R. 2017. Structure démographique et répartition spatiale des populations de Sclerocarya birrea (A. Rich.) Hochst. du secteur sahélien du Niger. Bois et Forêts des Tropiques, 333: 55-66.

Agbahoungba S, Assogbadjo AE, Chadare FJ, Idohou R, Salako VK, Agoyi EE, Kakaï RLG. 2016. Ecological diversity and conservation of wild edible fruit trees species in the Lama Forest Reserve in Benin. Bois et Forêts des Tropiques, 329(3): $\quad 353-365 . \quad$ DOI http://dx.doi.org/10.19182/bft2016.329.a 31312.

Agbogan A, Tozo K, Wala K, Bellefontaine R, Dourma M, Akpavi S, Woegan YA, Dimobe K, Akpagana K. 2015. Structure des populations de Sclerocarya birrea, Lannea microcarpa et Haematostaphis barteri au nord du Togo. Journal of Animal \& Plant Sciences, 25(2): 38713886.

Akoègninou A, Van der Burg W, Van der Maesen LJG, Adjakidje V, Essou J, Sinsin B, Yedomonhan H. 2006. Flore analytique $d u$ Bénin. Backhuys Publishers: Cotonou - Wageningen; p.1064. 
Akouehou GS, Goussanou CA, Idohou R, Dissou F, Azokpota P. 2014. Importance socioculturelle de Artocarpus altilis (Parkinson) Fosberg (Moraceae) au SudBénin. Journal of Applied Biosciences, 75(1): $\quad$ 6173-6182. DOI http://dx.doi.org/10.4314/jab.v75i1.5.

Ambé G-A. 2001. Les fruits sauvages comestibles des savanes guinéennes de Côte-d'Ivoire: état de la connaissance par une population locale, les Malinké. Biotechnologie, Agronomie Société et Environnement, 5(1): 43-58.

Assogba ODI, Salako KV, Fantodji B, Assédé ÉP, Assogbadjo AE, Chirwa PW. 2020. Does land use type impact the demographic and spatial structures of Adansonia digitata $\mathrm{L}$. in the Pendjari Biosphere Reserve in Northern Benin? Bois et Forets des Tropiques, 344: 59-72. DOI:

https://doi.org/10.19182/bft2019.344.a31 888.

Assogbadjo AE, Kakaï RLG, Sinsin B, Pelz D. 2010. Structure of Anogeissus leiocarpa Guill., Perr. natural stands in relation to anthropogenic pressure within WariMaro Forest Reserve in Benin. African Journal of Ecology, 48(3): 644-653.

Avana-Tientcheu MLA, Keouna S, Nguemo DD, Masdewel BM. 2019. Structure des peuplements et potentiel de domestication de Parkia biglobosa dans la région de Tandjilé-Ouest (Tchad). International Journal of Biological and Chemical Sciences, 13(1): 219-236. DOI https://dx.doi.org/10.4314/ijbcs.v13i1.19

Badiane M, Camara B, Ngom D, Diedhiou MAA. 2019. Perception communautaire des parcs agroforestiers traditionnels à Faidherbia albida (Del.) Chev. en Basse Casamance, Sénégal. Afrique Science, 15(1): 214-226.

Balna J, Gonne B, Madi OP, Abel T. 2015. Pratiques sylvicoles des pasteurs transhumants dans les agroforêts sèches $\mathrm{du}$ Nord Cameroun (Afrique centrale)[Silvicultural practices transhumant pastoralists in dry agroforests of North Cameroon (Central
Africa)]. International Journal of Innovation and Applied Studies, 13(3): 643-655.

Barmo S, Amani A, Soumana I, Ichaou A, Saley K, Mahamane A. 2019. Structure et diversité des parcs agroforestiers adjacents à la forêt protégée de Baban Rafi, Niger-Afrique de l'Ouest. Afrique Science, 15(2): 166-185.

Biaou S, Natta A, Dicko A, Kouagou M. 2016. Typologie des systèmes agroforestiers et leurs impacts sur la satisfaction des besoins des populations rurales au bénin. Bulletin de la Recherche Agronomique du Bénin, 43-56.

De Souza S. 2008. Flore du Benin: Nom des Plantes dans les Langues Nationales Beninoises. Imprimerie Tundé: Cotonou, Benin; p. 679.

Diedhiou M, Faye E, Ngom D, Toure MA. 2014. Identification et caractérisation floristiques des parcs agroforestiers du terroir insulaire de Mar Fafaco (Fatick, Sénégal). Journal of Applied Biosciences, 79 (1): 6855-6866.

Djodjouwin L, Kakai RG, Sinsin B. 2011. Caractérisation structurale des formations naturelles enrichies en essences forestières locales: cas des vertisols de la Lama (Benin). International Journal of Biological and Chemical Sciences, 5(4): 1628-1638.

Gbedomon R, Salako V, Chadare F, Kakaï RG, Assogbadjo A. 2016. Gendered motivation for home gardening and maintenance of agrobiodiversity: a case study in Benin, West Africa. Annales des Sciences Agronomiques, 20(2): 91-104.

Ginisty C. 2008. Mélanger les peuplements pour une forêt plus riche, voire plus résistante au changement climatique. Le thème de recherche BIOFOR. Cemagref, p. 5.

Hermans M, Akoègninou $\mathrm{A}$, van der Maesen J. 2004. Medicinal plants used to treat malaria in southern Benin. Economic Botany, 58(1): S239-S252.

Idohou R, Fandohan B, Salako VK, Kassa B, Gbèdomon RC, Yédomonhan H, Glèlè Kakaï RL, Assogbadjo AE. 2014. 
Biodiversity conservation in home gardens: traditional knowledge, use patterns and implications for management. International Journal of Biodiversity Science, Ecosystem Services \& Management, 10 (2): 89-100 DOI : https://doi.org/10.1080/21513732.2014.9 10554

INSAE 2016. Institut National de la Statistique et de l'Analyse Economique. Résultats provisoires du recensement général de la population et de l'habitation. (RGPH4). Cotonou, p. 39.

Kebenzikato AB, Wala K, Dourma M, Atakpama W, Dimobe K, Pereki H, Batawila K, Akpagana K. 2014. Distribution et structure des parcs à Adansonia digitata L.(baobab) au Togo (Afrique de l'Ouest). Afrique Science: Revue Internationale des Sciences et Technologie, 10(2): 434 - 449.

Koda DK, Adjossou K, Djego JG, Guelly KA. 2016. Diversité et usages des espèces fruitières des systèmes agroforestiers à caféiers du Plateau-Akposso au Togo. Afrique Science, 12(4): 113-119.

Mbaiyetom H, Tientcheu MLA, Ngankam MT, Taffo JBW. 2021. Diversité floristique et structure de la végétation ligneuse des parcs arborés de la zone soudanienne du Tchad. International Journal of Biological and Chemical Sciences, 15(1): 68-80.

DOI:

https://dx.doi.org/10.4314/ijbcs.v15i1.7

Ndong AT, Ndiaye O, Sagna MB, Diallo A, Galop D, Guisse A. 2015. Caractérisation de la végétation ligneuse sahélienne du Sénégal: cas du Ferlo. International Journal of Biological and Chemical Sciences, 9(6): 2582-2594.

Odebiyi J, Bada S, Awodoyin R, Oni P, Omoloye A. 2004. Population structure of Vitelaria paradoxa Gaertn. F. and Parkia biglobosa (Jacq.) Benth. in the agroforestry parklands of Nigerian humid Savanna. West African Journal of Applied Ecology, 5(1): 31-39.

ONU. 2016. Profil démographique de l'Afrique, Nations Unies. Commission économique pour l'Afrique, p.78.
Ouedraogo N, Tibiri A, Sawadogo R, Lompo M, Hay A, Koudou J, Dijoux M, Guissou I. 2011. Antioxidant anti-inflammatory and analgesic activities of aqueous extract From stem bark of Pterocarpus erinaceus Poir.(Fabaceae). Journal of Medicinal Plants Research, 5 (10): 2047-2053.

Pédelahore P. 2014. Systèmes agroforestiers à cacaoyers et transition capitaliste: l'exemple du Centre-Cameroun. Bois \& Forets des Tropiques, 321(321): 55-66.

Pereki H, Wala K, Thiel-Clemen T, Bessike MPB, Zida M, Dourma M, Batawila K, Akpagana K. 2013. Woody species diversity and important value indices in dense dry forests in Abdoulaye Wildlife Reserve (Togo, West Africa). International Journal of Biodiversity and Conservation, 5(6): 358-366. DOI: 10.5897/IJBC12.061

Phalan B, Balmford A, Green RE, Scharlemann JP. 2011. Minimising the harm to biodiversity of producing more food globally. Food Policy, 36: S62-S7. DOI:10.1016/j.foodpol.2010.11.008

Rabiou H, Bationo BA, Adjonou K, Kokutse AD, Mahamane A, Kokou K. 2017. Perception paysanne et importance socioculturelle et ethnobotanique de Pterocarpus erinaceus au Burkina faso et au Niger. Afrique Science, 13: 43-60.

Rigg J. 2009. A particular place? Laos and its incorporation into the development mainstream. Environment and planning A, 41(3): 703-721. DOI http://dx.doi.org/10.1068/a40260

Saj S, Jagoret P, Ngogue HT. 2013. Carbon storage and density dynamics of associated trees in three contrasting Theobroma cacao agroforests of Central Cameroon. Agroforestry Systems, 87(6): 1309-1320. DOI: 10.1007/s10457-0139639-4

Salako VK, Fandohan B, Kassa B, Assogbadjo $\mathrm{AE}$, Idohou AFR, Gbedomon RC, Chakeredza S, Dulloo ME, Kakaï RG. 2014. Home gardens: an assessment of their biodiversity and potential contribution to conservation of threatened species and crop wild relatives in Benin. 
Genetic Resources and Crop Evolution, 61(2): 313-330. DOI: 10.1007/s10722013-0035-8

Sardou J-D, Jean-Pierre D, Mutel M, Duchaufour H, Langlais C, Fernandes P, Alphonse M-E, Malézieux É. 2014. Évolution de la structure d'un système agroforestier en relation avec le cycle de vie familial: cas du jardin de case en Haïti. Bois \& Forets des Tropiques, 321: 7-20.

Sinsin B, Attignon S, Lachat T, Peveling R, Nagel P. 2003. La forêt de Lama au Bénin: un écosystème menacé sous la loupe. Opuscula Biogeographica Basileensia, 3: 1-32.

Suárez A, Williams-Linera G, Trejo C, ValdezHernández JI, Cetina-Alcalá VM, Vibrans H 2012. Local knowledge helps select species for forest restoration in a tropical dry forest of central Veracruz, Mexico. Agroforestry Systems, 85(1): 3555. DOI: 10.1007/s10457-011-9437-9

Toyi M, Eda F, Barima Y, Bamba I, Sinsin B. 2017. Dynamique paysagère de la Forêt Classée de la Lama au sud du Bénin. Tropicultura, 35(2): 1-14.

Traore L, Ouedraogo I, Ouedraogo A, Thiombiano A. 2011. Perceptions, usages et vulnérabilité des ressources végétales ligneuses dans le Sud-Ouest du Burkina Faso. International Journal of Biological and Chemical Sciences, 5 (1): 258-278.
Triplet P. 2016. Dictionnaire Encyclopédique de la Diversité Biologique et de la Conservation de la Nature (troisième édition). Patrick Triplet; p.1056.

Vodouhê F, Coulibaly O, Assogbadjo A, Sinsin B. 2008. Medicinal plant commercialization in Benin: an analysis of profit distribution equity across supply chain actors and its effect on the sustainable use of harvested species. Journal of Medicinal Plants Research, 2(11): $\quad 331-340 . \quad$ DOI: https://doi.org/10.5897/JMPR.9000867

Vodouhê F, Coulibaly O, Gauthier, Sinsin B. 2011.Traditional agroforestry systems and biodiversity conservation in Benin (West Africa). Agroforestry Systems, 82: $1-13$.

Wala K, Sinsin B, Guelly KA, Kokou K, Akpagana K. 2005. Typologie et structure des parcs agroforestiers dans la préfecture de Doufelgou (Togo). Science et Changements Planétaires/Sécheresse 16(3): 209-216.

Zerbo P, Millogo-Rasolodimby J, Guinko S, Van Damme P. 2014. Impact des tradipraticiens de santé dans la gestion durable des plantes médicinales au Burkina Faso: cas du Pays San. Pharmacopée et Médecine Traditionnelle Africaine, 17(1): 59-66. 